\title{
AGENDAMENTO DE DESCARGA DE MATÉRIAS PRIMAS NA ARCELORMITTAL AÇOS PLANOS AMÉRICA DO SUL*
}

\author{
Lidia Rangel Roveta \\ Marco Antonio Aburachid Tavares ${ }^{2}$ \\ Pablo Silva da Rocha ${ }^{3}$ \\ Daniel Costa Bezerra ${ }^{4}$
}

\section{Resumo}

O processo de aciaria da ArcelorMittal Aços Planos América do Sul tem um abastecimento de diário de Cal em torno de 420 ton. O processo de abastecimento deste produto, que consiste da programação, liberação de portaria e descarregamento não estava satisfatório, considerando o tempo de permanência de caminhões na portaria aguardando liberação e consequentemente custos com diárias e alimentação. Visando otimizar este processo, a partir do resultado da analise dos procedimentos de descarga, utilizado atualmente, foi implementado como plano de ação um sistema automatizado de agendamento de descarga de $\mathrm{Cal}$ que, após 15 dias de implantação resultou na redução de $66 \%$ no custo de pagamento de diária, além de previsão de ganho futuro em frete.

Palavras-chave: Agendamento; Descarga; Diárias; Frete.

\section{SCHEDULING SYSTEM OF UNLOADING PROCESS OF ROW MATERIAL AT ARCELORMITTAL FLAT CARBON SOUTH AMERICA}

\section{Abstract}

The steelmaking process of ArcelorMittal Flat Carbon South America has a daily supply of Lime about around 420 tonnes. The inbound process of this material which includes programming, entrance and unload wasn't satisfactory, due to a high waiting time and consequent overnight and meal costs. To optimize this process, from the results of the analysis of the discharge procedures currently used, has been implemented an automated Scheduling System that after 15 days of implementation resulted in $66 \%$ of cost reduction (overnight and meal), with forecast future gain in freight.

Keywords: Schedule system; Unload process; Overnight; Freight.

1 Tecnologia Mecânica, Pós-Graduação Engenharia de Materiais, MBA Gestão de Negócios, Especialista em Contratação, ArcelorMittal Aços Planos América do Sul, Serra, Espírito Santo, Brasil.

2 Engenharia de Produção, Analista de Planejamento Logístico, ArcelorMittal Brasil, Belo Horizonte, MG, Brasil.

3 Sistema de informação, Analista de processos de suprimentos, ArcelorMittal Aços Planos América do Sul, Serra, Espírito Santo, Brasil.

4 Ciência da Computação, Administração, Pós-Graduação Engenharia de produção, IFES, Analista de TI, ArcelorMittal Aços Planos América do Sul, Serra, Espírito Santo, Brasil. 


\section{INTRODUÇÃO}

Visando a melhoria no processo de descarga de Cal na ArcelorMittal Aços Planos América do Sul enxergamos uma possibilidade de contribuir para a otimização do fluxo de transporte de entrada de matérias primas - Cal, pois a grande quantidade de veículos para carregamento e descarga na portaria da ArcelorMittal Aços Planos América do Sul estava causando transtornos tais como:

- demora no atendimento dos motoristas;

- filas de entrada causando transtorno no atendimento da segurança patrimonial;

- custo da empresa diárias e alimentação;

- problema social com necessidade de mulheres e crianças viajarem com os motoristas; e

- risco de acidente dos motoristas e familiares que transitam entre as carretas

O projeto iniciou com o objetivo de:

- aumentar a eficiência no agendamento;

- reduzir tempo na descarga de materiais primas;

- Eliminar/Reduzir efeito dominó quando ocorre atraso (transportadora e operação);

- Melhorar Integração do planejamento da carga baseada na capacidade de descarga;

- Racionalizar uso de recursos de transporte.

Foram realizadas as seguintes ações:

- Analise da possibilidade de descarga 24h(operação / transportadora);

- Mapeamento do processo de operação, pontos de descarga da Cal / Calcário;

- Avaliação do processo de carregamento no fornecedor de Cal/Calcário.

\section{MATERIAIS E MÉTODOS}

A partir da análise do processo de descarga de Cal identificamos as seguintes rupturas:

\subsection{Portaria}

- Excesso de veículos aguardando para descarga;

- Elevado tempo de permanência pois os veículos não tinham previsão de entrada;

- Redução da agilidade no abastecimento;

- Controle manual do momento da chegada dos veículos;

- Quando os veículos eram liberados para entrar nem sempre era seguida a ordem de chegada;

- Ruídos de comunicação entre portaria e silos térreos;

- Aumento indireto do custo do frete, pois a falta de disponibilidade do veiculo fazia com que tivéssemos um numero maior de veiculo para atendimento.

\subsection{Silos Térreos}

- Falta de controle dos horários de chegada e descarga dos veículos na usina e consequentemente das diárias devidas, a planilha é enviada pela transportadora com os respectivos horários; 
- Falta de aderência da programação de carregamento com a descarga;

- Programação do carregamento não considerava a capacidade de descarga diária devido ao receio de falta de material;

Após esta avaliação foi identificado um sistema legado desenvolvido pela ArcelorMittal Sistemas que permite que a própria transportadora faça o agendamento após o faturamento no fornecedor de acordo com o planejamento de viagem além de permitir a parametrização de tempos de Descarga em nível de SKU (código de produto) e suporta a Gestão da Operação de Descarga por Evento.

O planejamento de viagem o sistema gera uma ordem de descarga que consta data e hora que o veiculo deverá se apresentar na usina.

Todas as etapas do processo são controlados desde a chegada até a saída do veiculo.(Figura 1)

Sistema de Agendamento controla todas Etapas do Processo (da chegada até saída do veículo)

\begin{tabular}{|c|c|c|c|c|}
\hline \multicolumn{2}{|c|}{ Check-in } & \multicolumn{3}{|c|}{ Tempo de Permanência e Descarga } \\
\hline Tolerância & Tempo Limite & Tempo de Entrada & Tempo de Descarga & Tempo de Saída \\
\hline
\end{tabular}

Figura 1. Etapas do processo

O funcionamento do sistema consiste em calcular conforme a parametrização a capacidade de descarga no ponto por:

1 - Ponto;

2 - GV (Grupo de vendas);

3 - Produto.

Conforme a parametrização na etapa de chamada poderá ser aplicada:

- Rotina de Liberação;

- Rotina de Expiração da Liberação;

- Rotina de Registro de Atraso;

- Liberação Manual.

Os pontos podem ser bloqueados não permitindo agendamento por período definido e devem ser justificados.

Em todas as etapas podem ser registradas as ocorrências conforme os motivos que levaram a atrasos, faltas, alterações de pontos, bloqueio de ponto, etc.

Vantagens observadas com a implantação do sistema de agendamento:

1 - Transportadora faz o agendamento via Web conforme planejamento de viagem e horário disponível;

2 - O sistema controla automaticamente a liberação dos veículos conforme agendamento;

3 - O operador do Silos Térreos tem uma visão diária da quantidade de veículos para descarga;

4 - Todas as etapas são registradas desde a chegada até a saída do veiculo tendo um controle mais efetivo desses horários;

5 - Maior controle nas cobranças de diárias conforme relatório das transportadoras;

6 - Os motoristas já sabem data e hora para descarga. 
Considerando:

- as funcionalidades do sistema;.

- avaliação da sistemática adotada nas usinas de longos;

- sistema amigável e com possibilidade de rollout para AMT.

Sugerimos a implantação do Sistema de agendamento de descarga da sendo proposto a categoria de Cal como projeto piloto com possibilidade de implantação futura em outros materiais.

Para implantação na ArcelorMittal Aços Planos América do Sul onde foram necessárias adequações e melhorias para atendimento das necessidades da planta.

\subsection{As fases do Projeto foram as seguintes:}

2.3.1 Sistema de implantação:

- Desenvolvimento do Sistema;

- Implantação

- Operação

- Suporte a Operação

- Gestão

- Comunicação

\subsubsection{Gestão dos tempos}

- Foco em melhoria continua do cumprimento de;

- Agendamentos

- Chegada pontual das transportadoras

- Descarga pontual pelas Unidades

- Melhoria de Produtividade das Unidades

- Revisão da Capacidade de Recebimento

\subsubsection{Ganho financeiro}

2.3.3.1 Desenvolver, validar e fazer a gestão para obter desconto de frete das transportadoras vinculado ao cumprimento do Agendamento.

\section{RESULTADOS E DISCUSSÃO}

Após o período de 15 dias de implantação observamos os seguintes resultados:

- Redução de veículos aguardando para descarga na portaria da AMT que era em media de 15 veículos para 2 veiculos no máximo;

- Aderência da transportadora à hora programada, ou seja veiculo chega na usina na hora programada conforme ordem de descarga;

- Redução do tempo de permanência de veículos para descarga de matériasprimas em 73\%;

- Redução no valor do pagamento de diárias (Figura 2);

- Possibilidade de ganhos na negociação de frete devido a maior disponibilidade do veiculo;

- Possibilidade de ganho na implantação deste sistema para agendamento de outros produtos, Ex: Materiais de suprimentos;

Gráfico de acompanhamento do resultado de ganho em diárias. 


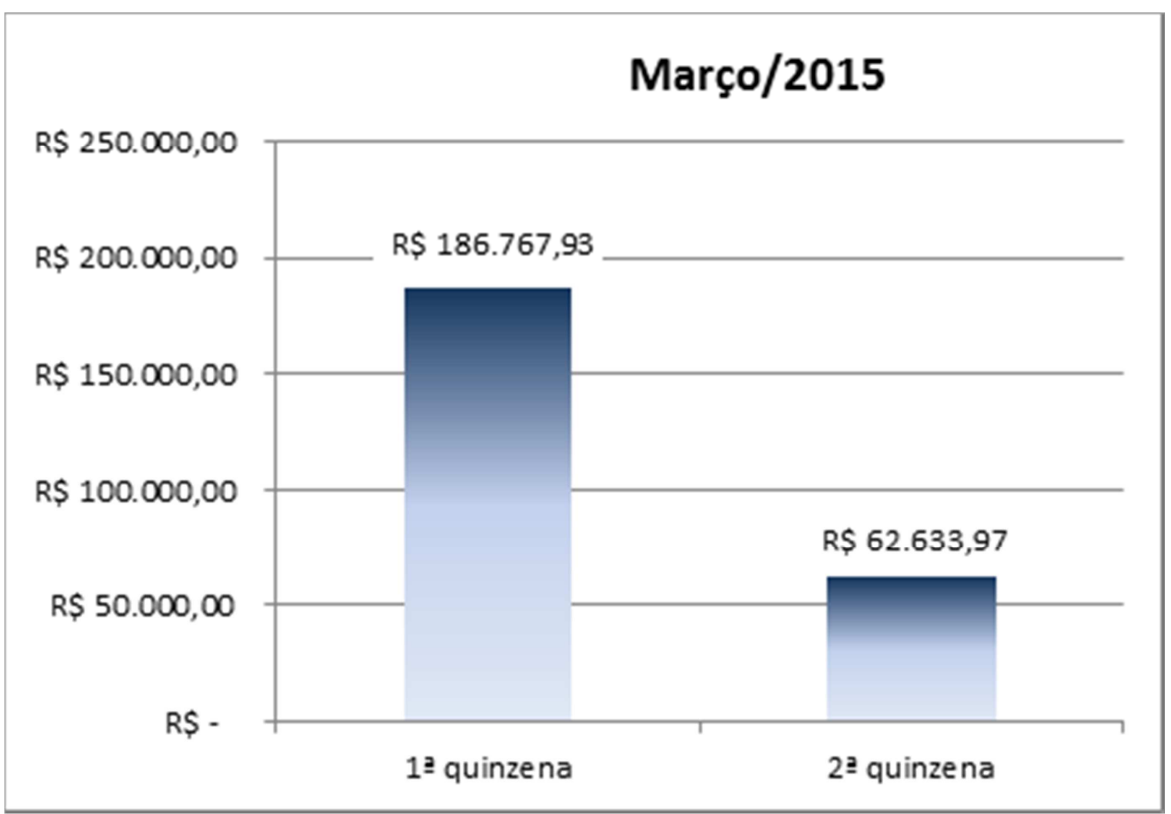

Figura 2. Comparação $1^{\circ}$ e $2^{\circ}$ quinzena mês de Março

O projeto de agendamento iniciou-se no dia 09/03.

1ํ fase: Período de 09 a 15/03. Testes e Operação Assistida AMS e Período para equipes e transportadoras se adaptarem ao Sistema. Foram feitos os agendamentos com os veículos que já se encontravam aguardando descarga

2ำ Fase: Período de 16 a 31/03: A Programação de carregamento foi enviada com antecedência conforme capacidade de descarga diária na AMT e os veículos agendados conforme janela e planejamento de viagem. $100 \%$ dos veículos agendados.

Conforme se pode ver no gráfico (figura 2) houve redução no valor devido de diária em $66 \%$ devendo-se a:

1.1 - Programação de carregamento mais aderente ao processo de produção;

1.2 - Implantação do agendamento;

1.3 - Veículos agendados no período de 24 horas conforme janelas de descarga.

\section{CONCLUSÃo}

O Sistema de Agendamento de descarga de Cal apresentou resultados satisfatórios uma vez que:

1. reduziu o tempo médio de espera em $73 \%$ - de 30 para 8 horas;

2. reduziu o custo com diárias pagas as transportadoras em $66 \%$;

3. reduziu a quantidade de veículos na portaria aguardando para descarga;

4. aumentou a satisfação dos motoristas que já chegam na usina com data e horários programados

5. aumentou a aderência da programação de carregamento e descarga. 